\title{
Transhiatal esophagectomy after previous right pneumonectomy
}

\author{
Jeffrey B. Velotta, MD, ${ }^{\mathrm{a}}$ Charles R. Vasquez, BS, ${ }^{\mathrm{b}}$ and David J. Sugarbaker, MD, ${ }^{\mathrm{a}}$ Boston, Mass, and
} Los Angeles, Calif

Esophagectomy after previous pneumonectomy is a rare occurrence, and the management is challenging. We present the case of a patient who had previously undergone a right pneumonectomy for T2aN0 squamous cell cancer and had development of esophageal adenocarcinoma, which was successfully managed with a transhiatal esophagectomy.

\section{CLINICAL SUMMARY}

A 64-year-old woman with a history of previous right pneumonectomy for T2aN0 squamous cell cancer 6 years,

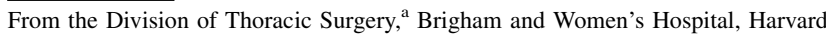
Medical School, Boston, Mass; and the David Geffen School of Medicine at UCLA, ${ }^{\mathrm{b}}$ Los Angeles, Calif.

Disclosures: Authors have nothing to disclose with regard to commercial support. Received for publication Feb 20, 2014; revisions received April 16, 2014; accepted for publication May 2, 2014; available ahead of print June 4, 2014.

Address for reprints: Jeffrey B. Velotta, MD, Division of Thoracic Surgery, Brigham and Women's Hospital, Harvard Medical School, 75 Francis St, Boston, MA 02115 (E-mail: jvelotta@partners.org).

J Thorac Cardiovasc Surg 2014;148:e150-2

$0022-5223 / \$ 36.00$

Copyright $($ c 2014 by The American Association for Thoracic Surgery http://dx.doi.org/10.1016/j.jtcvs.2014.05.005 treated with adjuvant chemotherapy (carboplatin/paclitaxel) was seen for a lower esophageal stricture at $35 \mathrm{~cm}$ after workup for dysphagia. Biopsy results were consistent with moderately differentiated adenocarcinoma. Endoscopic ultrasonography revealed a T3N0 lesion. Positron emission tomographic and computed tomographic (CT) scans showed a fludeoxyglucose F 18 (18F FDG)-avid mass in the lower esophagus with a standard uptake value of 7.8 and no metastases. After 5 cycles of leucovorin (INN folinic acid), fluorouracil, and oxaliplatin, repeated combination positron emission tomographic and computed tomographic (PET-CT) scans revealed decreased 18F FDG avidity. Neoadjuvant radiation therapy was not given because of concern regarding worsening of the extensive fibrosis already present from previous pneumonectomy. Preoperative cardiovascular workup was unremarkable, and pulmonary function tests showed an acceptable forced expiratory volume in 1 second (FEV 1) of $0.84 \mathrm{~L}$ ( $40 \%$ predicted). Pre-operative imaging findings are shown in Figure 1.

Our initial operative plan was to perform a modified McKeown 3-hole esophagectomy. We were unable to gain
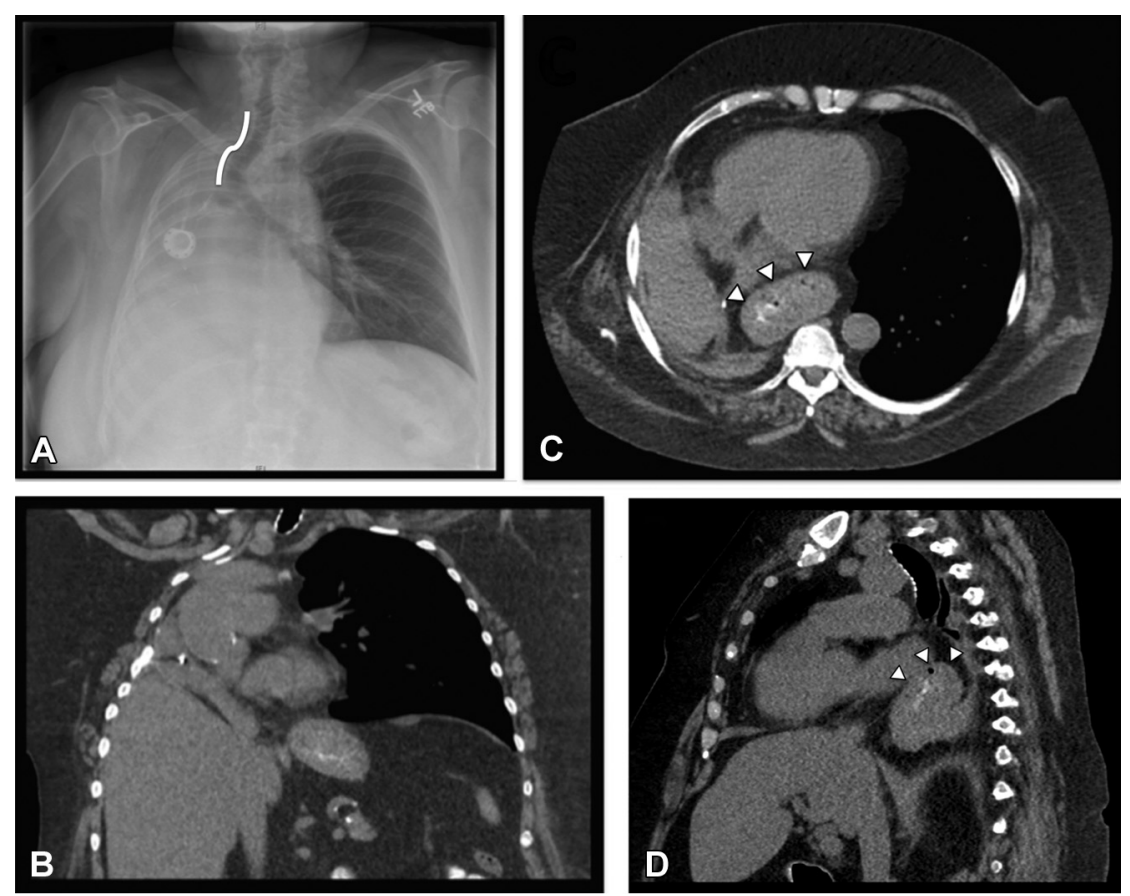

FIGURE 1. A preoperative upright chest radiograph (A) and a coronal-reconstructed computed tomographic scan (B) demonstrate the anatomic changes associated with previous right pneumonectomy, including significant tracheal deviation (white line). Both axial (C) and sagittal-reconstructed (D) computed tomographic scans also reveal the presence of a hiatal hernia (white arrowheads). 

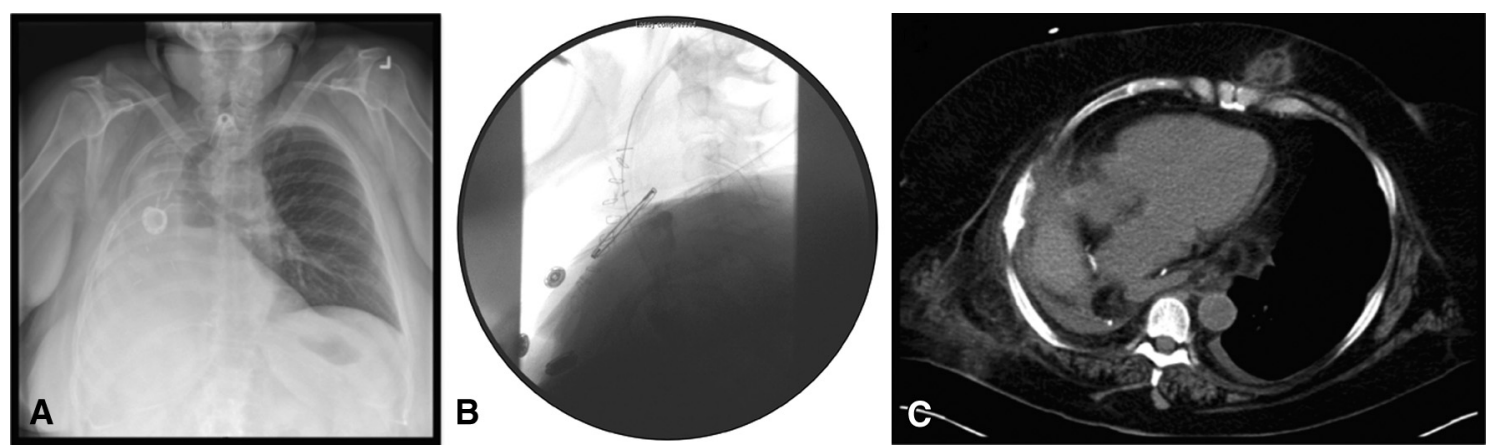

FIGURE 2. A, A postoperative upright chest radiograph demonstrates the interval placement of a tracheostomy and postsurgical changes. B, A sagittal image from thin barium esophagogram demonstrates the position of the new esophageal conduit. C, An axial computed tomographic scan also demonstrates this position.

entry into the right chest because of extensive thick, fibrotic scar tissue, however, and we therefore chose to convert to a transhiatal approach. An upper midline incision was performed, and no evidence of metastatic disease was seen. Esophageal mobilization and conduit preparation were performed in the standard fashion. The presence of an enlarged hiatus from a preexisting hiatal hernia facilitated our dissection by allowing simultaneous laparoscopic (10 mm, $30^{\circ}$; Olympus Corporation of the Americas, Center Valley, $\mathrm{Pa}$ ) placement and hand retraction in the mediastinum. Because of the postpneumonectomy mediastinal shift, we carried our dissection further, to the level of the bifurcation of the pulmonary artery. To facilitate adequate length for the cervical anastomosis, we performed a more extensive Kocher maneuver and further widened the hiatus to allow greater conduit mobilization. We were unable to free the periesophageal tissue completely because of dense adhesions, so our final specimen was stripped of some adventitial tissue but completely intact. Once the gastric conduit was prepared, the proximal esophagus was dissected with the aid of a standard mediastinoscope and thin suction irrigator. The specimen was brought up to the neck in a sterile arthroscopic bag. We then performed a side-to-side functional end-to-end stapled anastomosis. A 10F JacksonPratt drain was placed around the anastomosis posteriorly, and a nasogastric tube was inserted into the proximal portion of the gastric conduit. A $16 \mathrm{~F}$ red rubber jejunostomy tube was placed in left lower quadrant. Final pathologic examination was consistent with a yT3N1 moderately differentiated adenocarcinoma with lymphovascular but not perineural invasion, 1 of 9 lymph nodes positive for metastatic adenocarcinoma, and negative resection margins.

Postoperatively, the patient required a tracheostomy for hypercarbia secondary to secretions. A thin barium esophagogram showed no evidence of a leak and good emptying of the conduit (Figure 2). The patient was discharged to a rehabilitation facility on postoperative day 34 , and decannulation was performed 6 weeks later. The patient is now 6 months from surgery and is showing no evidence of recurrence, exercising without oxygen, and tolerating meals without aspiration or dysphagia.

\section{DISCUSSION}

The incidence of a newly diagnosed esophageal cancer after a previous diagnosis of lung cancer is exceedingly low, ranging from $0.05 \%$ to $0.5 \%{ }^{1}$ Furthermore, there are only 3 reported cases of esophagectomy being performed in a patient with previous pneumonectomy. ${ }^{2-4}$ To date, this is the first published report of a transhiatal esophagectomy after previous right pneumonectomy. Repeated entry into the thoracic cavity in a patient who has undergone pneumonectomy presents several challenges as a result of postoperative compensatory anatomic and physiologic changes. Anatomic changes include mediastinal shift toward the postpneumonectomy site, ipsilateral hemidiaphragm elevation, and hyperexpansion of the intact lung. ${ }^{2,3,5}$ Because of the anatomical changes and multiple potential approaches for esophagectomy, it is imperative that preoperative imaging studies be used to prepare a surgical plan. We recommend high-resolution CT to evaluate thoracic anatomy, in concert with positron emission tomography to assess for the presence of metastatic disease. Despite optimal CT scan imaging, as our case demonstrates, intraoperative findings such as dense adhesions may necessitate a change in operative approach. Both open thoracotomy and minimally invasive approaches have been reported previously. ${ }^{2,3}$ Transhiatal esophagectomy has the advantage of avoiding the postpneumonectomy space, which may have dense adhesions, as we encountered. Meticulous mediastinal dissection of the esophagus in the setting of extensive postpneumonectomy changes and mediastinal shifts is challenging but essential. We recommend the use of a mediastinoscope in the neck for better proximal esophageal mobilization and a laparoscope in addition to digital retraction in the hiatus for adequate exposure and lysis of adhesions. 


\section{References}

1. Fékété F, Sauvanet A, Kaisserian G, Jauffret B, Zouari K, Berthoux L, et al. Associated primary esophageal and lung carcinoma: a study of 39 patients. Ann Thorac Surg. 1994;58:837-42.

2. Reardon MJ, Estrera AL, Conklin LD, Reardon PR, Brunicardi FC, Beall AC. Esophagectomy after pneumonectomy: a surgical challenge. Ann Thorac Surg. 2000;69:286-8
3. Petri R, Brizzolari M, Sorretino M, Bassi F, Muzzi R, Zuccolo M. Minimally invasive esophagectomy in a previously pneumonectomized patient. J Laparoendosc Adv Surg Tech A. 2012;22:695-700.

4. Xu HC, Ye P, Bao FC, Pan H, Yang YH, Wang LM, et al. ECMO-assisted esophagectomy after pneumonectomy. Int J Artif Organs. 2013;36:259-62.

5. Stoller JK, Blackstone E, Pettersson G, Mihaljevic T. Coronary artery bypass graft and/ or valvular operations following prior pneumonectomy. Chest. 2007;132:295-301. 